\title{
Dust in the atmospheres of brown dwarfs and young planets: the effects of gravitational settling and convective overshoot
}

\author{
D. Homeier ${ }^{1}$, H.-G. Ludwig ${ }^{2}$, F. Allard ${ }^{3}$, \\ P. Hauschildt ${ }^{4}$ \& M. Dehn ${ }^{4}$ \\ ${ }^{1}$ Institut für Astrophysik, Universität Göttingen, Germany \\ ${ }^{2}$ Observatoire de Paris-Meudon, Paris, France \\ ${ }^{3}$ Centre de Recherche Astronomique de Lyon, École Normale Supérieure, Lyon, France \\ ${ }^{4}$ Hamburger Sternwarte, Universität Hamburg, Germany
}

\begin{abstract}
Dwarfs of the spectral types late-M, L and T span mass regimes from very-low-mass stars through brown dwarfs down to young planetary objects. They all show massive molecular line-blanketing and the condensation of refractory species with decreasing $T_{\text {eff }}$, leading to changes in chemical equilibrium composition and absorption due to dust grains. The spectral evolution from late-M through $\mathrm{L}$ to mid- and late- $\mathrm{T}$ classes is now understood as chiefly due to increasing amounts of condensates in the visible photosphere up to mid-L types, and the settling of dust clouds into deeper regions at the transition from $\mathrm{L}$ to $\mathrm{T}$, resulting in a depletion of condensable elements in the upper atmosphere. The ensuing photospheric cooling also drives a change in carbon chemistry leading to the hallmark methane absorption features of $\mathrm{T}$ dwarfs.

Recent observations of brown dwarfs in the L-T spectral sequence and model atmosphere calculations have shown that these changes in spectral features reveal differences in the efficiency of cloud removal that seem to be triggered by an additional parameter besides effective temperature. We present models describing the settling of particle clouds as an equilibrium process between condensation, gravitational sedimentation and convective and turbulent mixing, based on 3D-hydrodynamical simulations for the description of the velocity field. These calculations predict a strong dependence of the settling on gravity, and can therefore explain observed differences between the spectral energy distributions of brown dwarfs of equal luminosity as an effect of different mass. Based on these calculations we would expect even stronger deviations in the colours of young brown dwarfs of very low mass from those commonly observed in the field. Such predictions seem to be in aggreement with the first observations of candidate planetarymass objects, and would imply that extrasolar planets at very young ages have spectral energy distributions significantly different from previous models. Our models also predict characteristic time scales for the cloud formation processes that may be compared to observed variability in brown dwarfs.
\end{abstract}

\section{Discussion}

KäUFL: What are the typical rotational periods of BDs?

HoMeIER: Typical periods for fast rotators are similar to Jupiter or even below, down to $\sim 3 \mathrm{hrs}$, as inferred from rotational broadening and photometric variability, much longer periods exist as well, though. 